Service social

\title{
Travail social et champ sociojudiciaire : vers une contribution renouvelée?
}

\section{Isabelle F.-Dufour}

Volume 57, numéro 1, 2011

URI : https://id.erudit.org/iderudit/1006287ar

DOI : https://doi.org/10.7202/1006287ar

Aller au sommaire du numéro

Éditeur(s)

École de service social de l’Université Laval

ISSN

1708-1734 (numérique)

Découvrir la revue

Citer cet article

F.-Dufour, I. (2011). Travail social et champ sociojudiciaire : vers une contribution renouvelée? Service social, 57(1), 63-79.

https://doi.org/10.7202/1006287ar
Résumé de l'article

Le travail social a traditionnellement été très actif dans le champ sociojudiciaire. Or, depuis les années 80 , on constate que cette profession y est moins exercée. L'objectif de cet article est de débusquer les raisons qui ont provoqué cet exode et de mettre en exergue les principaux défis et enjeux contemporains reliés à la pratique du travail sociojudiciaire. Cette analyse critique permettra ensuite d'explorer le contexte actuel favorisant une contribution renouvelée de la discipline à ce champ de pratique. 


\title{
Travail social et champ sociojudiciaire : vers une contribution renouvelée?
}

Isabelle F.-Dufour

\section{RÉSUMÉ}

Le travail social a traditionnellement été très actif dans le champ sociojudiciaire. Or, depuis les années 80 , on constate que cette profession y est moins exercée. L'objectif de cet article est de débusquer les raisons qui ont provoqué cet exode et de mettre en exergue les principaux défis et enjeux contemporains reliés à la pratique du travail sociojudiciaire. Cette analyse critique permettra ensuite d'explorer le contexte actuel favorisant une contribution renouvelée de la discipline à ce champ de pratique.

Mots clés : Travail social; champ sociojudiciaire; enjeux; exode; réhabilitation; probation.

\begin{abstract}
Social work and criminal justice domain have always been traditionally related. But since the beginning of 1980's, there are less socials workers working in this field. This article aim to uncover the reasons that are related to this abandon and to explore the difficulties that social workers encounters today in this field. This socio historical analysis serves as a basis to explore the new opportunities that would make it possible for the social work discipline to reaffirm a strong presence in this field.
\end{abstract}

Keywords: Social work; criminal justice domain; difficulties; abandon; rehabilitation; probation.

\section{INTRODUCTION}

Le service social a été impliqué dans la théorisation et dans la pratique du système de justice pénale avant même que la profession émerge, soit depuis plus de cent ans (Ades et Spiro, 1985; Chaiklin, 2007), mais il existe peu d'écrits scientifiques permettant de retracer la contribution spécifique du travail social dans ce champ d'intervention (Roberts et Brownell, 1999). La relative absence de travailleurs sociaux œuvrant actuellement dans ce champ (Gumz, 2004) ou encore, la rareté des cours se rapportant à ce champ dans leur formation universitaire (Chaiklin, 2007) peuvent expliquer cette parcimonie. Toutefois, la principale difficulté de cet exercice semble être liée à la diversité des «formes » de travail social qui ont été pratiquées dans ce champ. En effet, on ne peut se référer au travail social sociojudiciaire comme s'il s'agissait d'une seule et même façon de concevoir les causes de la criminalité et la personne contrevenante $^{1}$, ou encore d'une seule et même manière d'intervenir auprès des personnes, des groupes ou des communautés. Par exemple, dans le champ sociojudiciaire, les travailleurs sociaux œuvrent notamment :

1. Désormais, seul le terme « contrevenant » sera utilisé. 
auprès des adultes ${ }^{2}$ dans les bureaux de probation, les centres résidentiels ou correctionnels communautaires, les organismes de réhabilitation sociale et les organismes qui œuvrent auprès des personnes qui ont des problèmes de santé mentale, de violence conjugale ou de toxicomanies pour autant que ces problèmes soient reliés à une sanction pénale (traduction libre, Roberts et Brownell, 1999, p. 362).

Le champ sociojudiciaire renvoie donc aux interventions destinées aux adultes qui ont été sentenciés et visant, habituellement, leur réhabilitation sociale. Encore une fois, considérant la diversité des institutions dans lesquelles ces activités se déroulent ainsi que le large spectre des personnes à qui des services sont offerts, et considérant aussi que le travail social dispose de trois principales méthodes d'intervention (individuelle, de groupe et communautaire), plusieurs «formes » de travail social se côtoient donc dans le champ sociojudiciaire. Ce qui semble néanmoins transversal aux diverses formes de travail social offert dans ce champ est le difficile exercice du maintien de l'équilibre entre l'aide et le contrôle (Gumz, 2004; Lancaster et Lumb, 2006; Normandeau, 1979; Ohlin, Piven et Pappenfort, 1956). Or, «lorsque les sociologues et les historiens soulignent le rôle des travailleurs sociaux dans le champ sociojudiciaire ils ont tendance à strictement mettre l'accent sur l'aspect contrôlant de cette profession » (Burford et Adams, 2004, p. 8).

Le premier objectif de cet article est de nuancer cette perception qui ne tient pas suffisamment compte des changements importants qui ont traversé la discipline du travail social selon les contextes historiques. Le second est de tenir compte de la particularité québécoise et canadienne de l'intervention sociale dans le champ sociojudiciaire. Étant donné toutefois le petit nombre d'écrits ${ }^{3}$ spécifiques à cette région géographique, les propos de l'article ont dû être appuyés par la littérature scientifique provenant d'autres pays où l'on a observé une progression historique similaire. Cette reconstitution historique «élargie » permet ainsi : 1) de mettre en exergue la contribution spécifique du travail social au champ sociojudiciaire; 2) de mieux saisir les enjeux qui ont poussé les travailleurs sociaux à abandonner ce champ; pour finalement 3) souligner comment la discipline pourrait offrir une contribution renouvelée à ce champ de pratique. Mais avant, il semble opportun de souligner ce qu'on entend par « travail social » :

La profession d'assistant social ou de travailleur social cherche à promouvoir le changement social, la résolution de problèmes dans le contexte des relations humaines et la capacité et la libération des personnes afin d'améliorer le bien-être général. Grâce à l'utilisation des théories du comportement et des systèmes sociaux, le travail social intervient au point de rencontre entre les personnes et leur environnement. Les principes des droits de l'homme et de la justice sociale sont

2. Les interventions destinées aux adolescents appartiennent au champ de la justice juvénile plutôt qu'au champ sociojudiciaire (Maschi, Violette, Scotto Rosato et Ristow, 2009, p. 232).

3. Entre septembre 2007 et juin 2011, la recherche documentaire a couvert les périodiques, livres, thèses et mémoires ainsi que les rapports produits par les agences sociales et par les ministères de la Sécurité publique du Québec et de la Justice du Canada. Des 109 écrits recensés portant spécifiquement sur le travail social dans le champ sociojudiciaire, uniquement 15 proviennent du Canada. 
fondamentaux pour la profession (Fédération internationale des travailleurs sociaux, 2000).

\section{L'ÉMERGENCE D'UNE DISCIPLINE}

Jusqu'à la fin du $X V I^{e}{ }^{e}$ siècle, les Canadiens ont le sentiment que la meilleure façon de se prémunir contre la criminalité est de bâtir de fortes communautés centrées sur l'Église et la famille (Ekstedt et Griffiths, 1988). Or dès le début du XIX ${ }^{\mathrm{e}}$ siècle, on assiste à l'effritement des petites communautés qui assumaient jusque-là les soins, ainsi que le contrôle des populations dépendantes ou déviantes (Ekstedt et Griffiths, 1988). Placé devant l'évidence que les familles et la communauté ne peuvent désormais assurer la cohésion et le contrôle social, l'État crée «des institutions de contrôle et de redressement moral (la trilogie de la prison, l'asile et l'institution charitable) se voulant aptes tant à resocialiser les délinquants et les pauvres valides qu'à prendre en charge les cas extrêmes de dénuement, notamment les inaptes au travail (malades pauvres, enfants, vieillards et invalides) » (Aranguiz et Fecteau 1998, p. 85). Selon plusieurs auteurs (Cohen, 1985; Foucault, 1975; Garland, 1985), cette période historique correspond à l'émergence d'une nouvelle forme de régulation sociale où le pouvoir ne s'affirme plus dans sa forme purement répressive, mais plutôt par la légitimation issue des connaissances acquises par la surveillance, la normalisation et l'examen (Foucault, 1975). C'est dans cette période où l'on recourt de plus en plus à l'enfermement que naissent la médecine, la psychiatrie et le travail social, disciplines qui permettront de construire, de modifier et d'opérationnaliser les systèmes de classement qui serviront à identifier et à « diviser » les populations (Parton, 1994). Pour le travailleur social de l'époque, il s'agit de distinguer le pauvre méritant du pauvre non méritant, soit de «diviser » les populations qui méritent de l'aide et celles qui doivent leur situation à la paresse ou au vice, et qui, elles, feront l'objet de répressions (Mayer, 2002).

Puis, progressivement, la pauvreté devient «sociale », c'est-à-dire qu'elle n'est plus comprise strictement comme une faiblesse morale, mais comme l'amalgame des initiatives personelles et la bienveillance de l'état (Parton, 1994). L'émergence du caractère social de la pauvreté impose désormais une nouvelle lecture des problèmes sociaux : on les saisit dès lors comme étant enchevêtrés entre les sphères publique et privée, et c'est précisément dans cet espace que choisit de s'inscrire le travail social (Donzelot, 1980). Pour s'immiscer dans cet espace, les premiers travailleurs sociaux œuvrant dans les Charity Organisation Societies (COS) vont pénétrer le domicile des pauvres en échange de ressources financières ou matérielles et ils tenteront, de l'intérieur, de favoriser des changements de comportements ou de mode de vie (Parton, 1994). Dès 1917, ces pratiques seront consacrées dans le livre de Mary Richmond sous le terme de «casework » ou «diagnostic social ». Le « casework» repose sur l'idée que l'accumulation d'une large quantité d'informations sur un individu dans son contexte social permet d'établir un diagnostic qui sera suivi d'un traitement approprié (Hussey et Duffee, 1980).

Le « casework » est associé à la probation dès 1918 lorsque William Cooley introduit cette méthode d'intervention à la Catholic Charities Probation of New York City (Hussey et Duffee, 1980). La probation de l'époque consiste à éviter qu'un contrevenant soit incarcéré sous 
réserve qu'il demeure sous la surveillance d'une personne qui s'assure de son bon comportement dans la communauté en agissant en «ami et conseiller » (Commission royale d'enquête sur le système pénal du Canada (Archambault) ${ }^{4}, 1938$, p. 225). Le premier agent de probation connu en Amérique du Nord est John Augustus (1784-1859), un cordonnier de Boston qui a accueilli chez lui plus de 2000 contrevenants (Hussey et Duffee, 1980). Au Canada, bien qu'il faille attendre jusqu'en 1927 pour que la probation soit officiellement inscrite au Code criminel, elle est utilisée dès 1892 (Archambault, 1938). Par exemple, entre 1921 et 1938, 9000 personnes ont été placées en probation en Ontario (Archambault, 1938). Puis, dès la création officielle du rôle d'agent de probation au Canada, on choisira la méthode d'intervention du « casework » pour la réhabilitation des personnes contrevenantes (Hussey et Duffee, 1980). Et puisque le «casework » a l'objectif de «réhabiliter tant le pauvre que le criminel » (Hussey et Duffee, 1980, p. 206), de nombreux travailleurs sociaux feront leur entrée dans le champ sociojudiciaire. En plus de la probation, la présence des travailleurs sociaux s'étendra aux agences sociales orientées spécifiquement vers la réhabilitation sociale des contrevenants $^{5}$ qui seront créées grâce à la Loi de l'assistance publique de 1921 qui reconnaîtra l'importance de « l'assistance hors les murs ».

Au moment où se spécifient les interventions « centrées sur les troubles personnels, les intérêts privés, la réhabilitation et l'actualisation de soi » (Franklin, 1990, p. 59), on note également la consolidation des groupes de défense des droits des personnes détenues. La Prisoners'Aid Association of Montreal, formée au début du siècle par le révérend J. D. Borthwick, est jumelée à la Canadian Prisoners' Welfare Association en 1930, et plus tard à la John Howard Society of Quebec (Kidman, 1947). Ces groupes « font la promotion du bienêtre des prisonniers et de leurs proches, visent l'amélioration des conditions de vie des prisonniers et la réforme du droit pénal et de ses procédures pour qu'il s'ajuste aux principes modernes des sciences sociales et humaines » (Kidman, 1947, p. 17). Leurs interventions se rapportent donc plus « aux besoins collectifs, à l'action sociale et à la réforme sociale » (Franklin, 1990, p. 59), et elles constituent les premiers pas du travail social communautaire dans le champ sociojudiciaire. C'est néanmoins sur le plan de l'intervention individuelle que le travail social s'est le plus illustré dans le champ sociojudiciaire pendant cette période historique. La Commission royale d'enquête sur le système pénal du Canada de 1938 (rapport Archambault) mentionne comment la probation est tributaire de cette profession :

Le succès de tout système de probation dépendra de la qualité du personnel qu'on lui attribuera. Ces officiers devront faire l'objet d'un recrutement soigné dans le rang des travailleurs sociaux les mieux formés [...]. Ces personnes devront être d'une infinie patience tout en pouvant exercer, au besoin, une discipline ferme (Archambault, 1938, p. 230).

Le rapport avance également (1938, p. 230) « qu'un bon agent de probation devrait faire réaliser au contrevenant que les conditions associées à sa liberté, plutôt que dirigées vers la

4. Désormais « Archambault ».

5. Comme la Société d'orientation et de réhabilitation sociale créée à Montréal en 1945 et le Service de réadaptation sociale fondé en 1946 à Québec (Poupart, 2004). 
punition, sont mises en place pour l'aider à acquérir de bonnes habitudes et pour développer un mode de vie plus ordonné et discipliné ». Ce rapport légitime donc les interventions des travailleurs sociaux, en plus de reconnaître que ces derniers sont les mieux placés pour faciliter la réhabilitation des contrevenants. Or, comme on le voit au cours de la prochaine période historique, le fait d'être associé aussi intimement à la réhabilitation ne sera pas uniquement salutaire à la discipline du travail social.

\section{LA PARADOXALE CONSOLIDATION ET REMISE EN QUESTION DE LA PROFESSION}

Si la discipline du travail social a contribué à l'émergence du « social », elle a rapidement investi cet espace où le privé et le social interagissent (Parton, 1994). II n'est toutefois pas facile d'occuper cet espace puisque le mandat du travail social « doit demeurer suffisamment large pour que diverses problématiques puissent être interprétées, mais également pour qu'elles soient résolues de diverses façons »(Parton, 1994, p. 18). De plus, puisque le travail social occupe cet espace entre le respectable et le déviant, entre ceux qui ont le pouvoir et ceux qui en sont exclus, non seulement il est le médiateur entre ceux qui sont «potentiellement ou réellement exclus, et le reste de la société »(Parton, 1994, p. 18), mais en plus, il a le devoir d'identifier «le caractère caché des individus, ce qu'il y a d'essentiellement bon, de vrai, d'authentique et de non aliéné en eux » (Philip, 1979, p. 99). C'est donc parce que le travail social agit à l'intersection de deux univers (privé/public, respectable/déviant, visible/caché, etc.); parce que ses méthodes d'intervention peuvent s'adresser plus spécifiquement ou simultanément à l'individu ou à sa communauté; et enfin parce qu'il peut servir à la fois de régulateur ou d'intégrateur social que Parton $(1994$, p.18) le qualifie « d'ambigu, incertain et contesté ». C'est ce caractère labile et mouvant de la profession qui expliquerait qu'on observe à la fois la consolidation et la remise en question du travail social dans le champ sociojudiciaire durant cette seconde période historique.

Sur le plan de la consolidation, notons que le travail social va investir le champ sociojudiciaire jusqu'à la fin des années 1960, au point qu'il deviendra « la seule profession à être présente dans toutes les parties du système de justice pénale » (Treger, 1983, p. 8). On aura même le sentiment que le « casework » constitue l'unique approche pour la réadaptation des marginaux, des délinquants et des prisonniers (Laplante, 1989). Deux facteurs sont particulièrement favorables à l'appropriation de ce champ : la création de l'État-providence et une forte croyance en la réhabilitation. L'État-providence s'est structuré autour du désir de réguler l'économie à partir d'interventions judicieuses de l'État pour favoriser un haut niveau d'activité économique et le plein emploi et d'autre part par la répartition des risques sociaux selon les principes actuariels, de façon à réduire les inégalités sociales (Parton, 1994). L'étatprovidence a donc permis d'espérer que l'universalité des services sociaux pourrait combler les inégalités sociales et favoriser la justice sociale. Cet optimisme caractérisait également le travail social de l'époque qui se croyait en mesure d'assister l'État dans son souhait de venir à bout des problèmes sociaux (Parton, 1994). Dans le champ sociojudiciaire, cet optimisme est accentué par une forte croyance en la réhabilitation (Tregger, 1983) : désormais, les classes « dangereuses » qui ont toujours été au centre des interventions sociales vont cesser d'exister, car «les dépravés sont en réalité des dépossédés ou des négligés et ils pourront être réhabilités »(Parton, 1994, p. 22). Dans un tel climat de confiance et d'optimisme, «les 
ambiguïtés, les tensions et les incertitudes [qui étaient au cœur de la profession] ont été submergées » (Parton, 1994, p. 22), et cela, jusqu'au début des années 1970.

Les années 1970 marquent toutefois les balbutiements du néolibéralisme et se caractérisent par une critique sévère de l'État-providence qui est perçu comme générant de trop grandes dépenses, n'incitant pas à l'emploi et créant une masse sans cesse grandissante de travailleurs improductifs (Parton, 1994). Pire encore,

\begin{abstract}
[l]'État-providence tel qu'il prend forme par l'action de ses nouveaux experts, exemplifié ultimement par les travailleurs sociaux, encourage une attitude douce et permissive envers les déviants, les inconscients ainsi que les violents ou potentiellement violents : la traditionnelle classe dangereuse. La permissivité a non seulement drainé les ressources, mais a également érodé la notion de responsabilité (traduction libre, Parton, 1994, p. 23).
\end{abstract}

Or la question de la responsabilité dans le champ sociojudiciaire demeure un enjeu majeur. Par exemple, si l'on conçoit que la criminalité est la résultante d'une société insuffisamment inclusive, on attribuera une part négligeable de la responsabilité des délits au contrevenant. À l'inverse, on lui attribuera l'entière responsabilité de ses actes si l'on juge que la criminalité est avant tout un choix rationnel. C'est pourquoi les années 1970, qui sont le point de rencontre de ces deux perspectives opposées, vont être caractérisées par des critiques venant de toutes parts. Ceux qui attribuent la responsabilité des délits au système social remettent en question le trop grand pouvoir discrétionnaire accordé aux experts du traitement et de la réhabilitation. Ils décrient l'utilisation des sentences à durée indéterminée où la peine n'échoit que lorsque l'expert juge que le contrevenant est « guéri » et ils mettent en exergue le rôle oppressif du système de justice pénale (Landreville, 1986; Normandeau, 1979). À l'opposé, ceux qui abondent en faveur du choix rationnel souhaitent des peines plus punitives. Ils croient que les traitements offerts aux contrevenants nuisent à l'objectif de dissuasion des peines et avancent que les peines trop douces expliquent la montée du taux de criminalité (Hussey et Duffee, 1980). Ils s'appuient, entre autres, sur l'article controversé de Martinson (1974), lequel, au terme de l'évaluation de 231 programmes de réhabilitation des contrevenants, conclut que lorsqu'il est question de réhabiliter les contrevenants, « rien ne marche » (« nothing works ») ${ }^{6}$. Cela donne lieu à une nouvelle façon de concevoir le traitement réhabilitatif au Canada; désormais,

[l]es établissements correctionnels doivent offrir des opportunités aux contrevenants [...]. Le principe des opportunités est basé sur la croyance que le contrevenant est ultimement responsable de ses comportements [...], qu'il est jugé et sentencié en fonction de son geste criminel, et non sur la base d'un désordre de la personnalité sous-jacent ou en raison de privations socio-économiques (traduction libre, cité dans Ekstedt et Griffiths, 1988, p. 56).

6. Martinson s'est enlevé la vie peu après s'être rétracté (Maruna, 2001, p. 166). 
En adoptant cette perspective, la réhabilitation sera désormais placée au second plan des orientations pénales et du même coup, le système de justice pénale tendra également à se dissocier des travailleurs sociaux (Hussey et Duffee, 1980). D'autant plus qu'on avançait que le « casework » ne parvenait pas à réduire la récidive ${ }^{7}$, et pouvait même la favoriser ${ }^{8}$. C'est à ce moment que l'approche cognitive comportementale, qui provient de la psychologie, viendra se substituer au « casework» des travailleurs sociaux car :

Contrairement au « casework » qui fait appel la l'intuition [insights] et à la qualité de la relation thérapeutique, l'approche cognitive comportementale met l'accent sur les comportements observables et manipule systématiquement l'environnement de façon à modifier ces comportements (Hussey et Duffee, 1980, p. 219-220).

Dans le champ sociojudiciaire, cette approche thérapeutique repose sur la théorie de la «mesure du risque » qui avance que les attitudes, les comportements et les valeurs des contrevenants sont appris et renforcés, et par conséquent, qu'ils peuvent être remodelés à l'aide de programmes d'aide basés sur les notions de risque, de besoin et de réceptivité (Goff, 2003). La notion de risque veut que les programmes d'aide soient d'intensité égale à celle du risque de récidive mesuré chez le contrevenant. Selon le deuxième principe, il faut tenir compte des « besoins » qui découlent des traits criminogènes propres du contrevenant. Les besoins reconnus étant :

Changer les attitudes antisociales, changer les émotions antisociales, réduire les fréquentations avec des personnes antisociales, promouvoir l'expression de l'affection et de la communication, promouvoir l'automonitorage et l'autosupervision, promouvoir l'identification et l'association avec des personnes non criminelles ou favoriser le contrôle de soi et la résolution de problèmes (traduction libre, Andrews, 1989, p. 16).

Finalement, le principe de réceptivité repose sur l'idée qu'il existe plus d'une façon d'acquérir de nouvelles aptitudes, et qu'il faut que le programme s'adapte au mode d'apprentissage du contrevenant. Un dernier principe implicite de ces programmes d'aide est l'obligation de respecter scrupuleusement toutes les étapes du programme pour qu'il soit efficace pour réduire la récidive. Lorsque ces conditions sont respectées, les évaluations des programmes d'aide basés sur l'approche cognitive comportementale (CC) sont très positives. Par exemple, l'étude d'Andrews, Bonta, Gendreau et Cullen (1990) montre que ces programmes peuvent réduire de $40 \%$ le risque de commettre une récidive, ce qui sera par la suite appuyé par de nombreuses méta-analyses (Andrews et Bonta, 1998; Gendreau, Little et Groggin, 1996; Lipton, Thornton, McGuire, Porporino et Hollin, 2000). C'est ce qui explique que ces programmes d'aide furent implantés rapidement dans la plupart des pays du Commonwealth, certains y voyant un mouvement presque évangélique (Mair, 1997).

7. Le fait de commettre une nouvelle infraction à la suite d'un suivi pénal et d'en être reconnu coupable.

8. Des vérifications récentes des études les plus défavorables au « casework » (la Cambridge-Somerville Study et I'Impact study) avancent plutôt que les effets observés sont minimaux et pas très convaincants (Burnett, 2002; 2004). 
Ce qui est intéressant dans ce modèle de gestion du risque, c'est qu'il introduit une toute nouvelle conception des responsabilités et de la réhabilitation sociale. Sous l'État-providence, le fait d'appartenir à la communauté était suffisant pour assurer au citoyen des droits et des obligations. La réhabilitation était alors la responsabilité de la société. Or ce modèle tient le contrevenant responsable de ses actes, et c'est donc à l'individu que revient la tâche de sa propre transformation. Si le processus échoue, c'est également à lui d'en porter la responsabilité (Kaminski, 2006). La réhabilitation ne s'est donc pas éteinte avec l'arrivée de ces programmes, mais elle a pris une tout autre forme. C'est néanmoins la continuité de cette idée d'une possible transformation de la personne contrevenante (ou d'assistance à sa transformation) qui assure le caractère hybride du système pénal canadien, lequel n'a pas basculé dans la punition stricte des contrevenants comme aux États-Unis (Mauruto et HannahMoffat, 2006). Même si les peines sont également plus punitives au Canada, « les professions du social ont conservé de robustes théories et des méthodes d'intervention réhabilitatives qui ont constitué des remparts efficaces contre les excès du tournant punitif » (O'Malley, 2004, p. 332). C'est cette résistance au mouvement punitif qui a assuré un espace où peuvent encore agir les intervenants sociaux.

S'il faut se battre pour assurer la préservation de la réhabilitation au sein du pénal, on assiste par ailleurs à une redistribution des responsabilités sociales entre l'État, le marché, le tiers secteur ${ }^{9}$ et le secteur informel au début des années 1980, surtout sur le plan de la santé et des services sociaux (Vaillancourt, 2002). Si une part de cette redistribution revient au néolibéralisme, elle a aussi été paradoxalement encouragée par les critiques venant « du milieu universitaire, des féministes, des socialistes, des minorités ethniques ou d'autres groupes d'intérêts » (Parton, 1994, p. 25). Tous souhaitent: 1) qu'une plus grande part des services sociaux soit offerte par les organismes communautaires, les agences privées ou les organisations bénévoles; 2) que les services soient décentralisés de façon à favoriser l'organisation communautaire; 3) que les rapports hiérarchiques soient horizontaux et sous forme contractuelle; et finalement 4) qu'on place le « consommateur » des services au centre des prises de décision (Parton, 1994). À l'origine, on souhaitait ce virage principalement pour offrir plus de souplesse aux interventions sociales, ainsi que pour favoriser la démocratie (Favreau, 2000, p. 43), mais le « virage communautaire » dans le champ sociojudiciaire a plutôt eu l'effet de réorienter l'intervention vers la gestion de cas et d'offrir une réponse plus fragmentée et davantage dirigée vers les symptômes que vers les personnes (Dufresne et Hastings, 2003). Pour les intervenants sociaux du champ sociojudiciaire communautaire, ce virage est interprété comme une « instrumentalisation du communautaire dans une politique de rationalisation budgétaire »(Dufresne et Hastings, 2003, p. 425), où les bailleurs de fonds conservent un «contrôle non négligeable sur la nature et la qualité des services offerts » (Dufresne et Hasting, 2003, p. 419).

Certains auteurs avancent toutefois que les organismes communautaires du champ sociojudiciaire n'ont pas été « victimes » de cette orientation, mais plutôt qu'ils auraient adopté

9. Le tiers secteur « est formé de l'ensemble des entreprises sociales de production de biens et services, des organismes communautaires autonomes, des coopératives et des mouvements de défense des droits » (Vaillancourt, 2002, p. 19). 
volontairement une attitude plus formaliste, plus procédurale et plus normée afin de renforcer leur position professionnelle (Bradt et Bouverne-De Bie, 2009a). Cela pouvant s'expliquer par la tension maintes fois documentée entre le «travail social individuel » et «le travail social communautaire » au sein de la discipline (voir Franklin, 1990). D'ailleurs, le déplacement des contrevenants vers «le communautaire » aura une influence importante sur la reconfiguration du rôle de l'agent de probation qui œuvre dans une perspective individuelle. Désormais, plutôt que « d'aider, d'assister et de surveiller », il devient un « courtier de ressources » (« broker »). Selon ce modèle, l'expert est sur le terrain et le rôle de l'agent de probation est d'accompagner son « client » vers les ressources existantes (Normandeau, 1979). N'étant plus le responsable de «l'aide et de l'assistance », il ne lui reste que le rôle de surveillance, ce qui explique qu'on l'a perçu progressivement comme un agent de contrôle (Normandeau, 1979).

Cette reconfiguration du rôle des travailleurs sociaux, tant sur le plan de l'intervention individuelle que communautaire, aura des effets profonds sur la présence de travailleurs sociaux dans le champ sociojudiciaire. Ce champ qui attirait près de $12 \%$ des travailleurs sociaux étatsuniens en 1951 verra cette proportion diminuer à seulement $1 \%$ en 1991 (Reamur, 2004, p. 219) ${ }^{10}$. Plusieurs facteurs globaux peuvent expliquer cette désertion. Un premier facteur est le sentiment que les interventions dans ce champ contredisent désormais les valeurs de justice sociale, d'autodétermination et de respect de la dignité de la personne qui sont au cœur de leur profession (Agllias, 2004; Barry, 2007). Un deuxième facteur renvoie à la déprofessionnalisation associée à l'obligation « d'appliquer » de façon stricte les programmes de réhabilitation issus des courants cognitifs comportementaux (Lancaster et Lumb, 2006). Un troisième facteur renvoie au manque de formation accessible aux intervenants sociaux qui veulent œuvrer dans ce champ (Chaiklin, 2007). Un quatrième facteur renvoie au sentiment d'être aliéné de «la base » ou encore de ne pas être reconnu par le corps disciplinaire lorsqu'on œuvre dans ce champ (Roberts et Brownell, 1999). Puis, sur un plan global, les travailleurs sociaux nouvellement formés « s'éloignent des champs de pratique où ils ont à œuvrer auprès de personnes marginalisées ou à risque de le devenir comme celles rencontrées dans le champ sociojudiciaire. Ils se dirigent plutôt vers des emplois mieux payés et dont le prestige est plus élevé »(Gumz, 2004, p. 458). Devant l'ensemble de ces facteurs, certains auteurs ont conclu que les travailleurs sociaux avaient été marginalisés du champ sociojudiciaire (Burford et Adams, 2004). D'autres avancent qu'il s'agit d'un retrait volontaire de leur part (Young et LoMonaco, 2001). Certains, enfin, croient qu'ils ont éprouvé des problèmes liés à leur identité professionnelle qui les ont fait abandonner ce champ au profit d'autres professions telles que la criminologie, la psychologie, le droit ou même les sciences infirmières (Roberts et Brownell, 1999). Le constat de cette reconstruction historique est plutôt que ces facteurs ont été vécus simultanément et qu'ensemble ils ont provoqué la mésalliance entre le travail social et le champ sociojudiciaire. Or il importe de dépasser ce constat afin d'envisager comment le travail social pourrait apporter une contribution renouvelée en matière d'approche à l'égard des contrevenants qui sont les individus les plus marginalisés de nos sociétés (Agllias, 2004; Gumz, 2004).

10. II n'a pas été possible de trouver des statistiques pour le Canada, étant donné que les travailleurs sociaux relèvent plus souvent de leur association provinciale et que leur adhésion est volontaire. 


\section{VERS UNE CONTRIBUTION RENOUVELÉE?}

Depuis environ vingt ans, on avance que nos sociétés sont passées de la modernité à la postmodernité. Ce qui distingue ces deux phases, c'est que dans la seconde, on a renoncé à l'idée qu'il existe une explication universelle du monde social et de la « nature humaine », pour affirmer plutôt que la vie sociale est diverse, flexible, plurielle, contingente et incertaine. Ce qui devient alors central dans les sociétés postmodernes n'est pas de valider l'existence de « la » société, mais plutôt de saisir le sens que chacun lui accorde (Hugman, 2003). Cela a néanmoins des désavantages, car si l'on nie l'existence de «l'universel », il y a un risque de sombrer dans un relativisme et un subjectivisme absolus qui rendent difficile l'identification de ce qui est « vrai » (Hugman, 2003). Or, paradoxalement, dans la même période, on a reconnu que la pauvreté était « vraie » et que les personnes provenant des minorités ethniques étaient « vraiment » plus souvent emprisonnées que les autres (Hugman, 2003). Pour saisir à la fois le sens et le vrai, la discipline du travail social s'est dotée d'un cadre théorique «postmoderne critique » qui reconnaît le caractère diversifié et flexible du « sens » tout en admettant qu'il puisse y avoir un discours éthique qui soit partagé plutôt qu'individuel (Hugman, 2003). Cela rend donc possible l'opposition entre le sens individuel accordé à une situation et le discours éthique pluriel qui émane du monde social, et lorsque cette situation se produit le travailleur social doit faire des choix (Hugman, 2003). Or la notion de choix est très réduite dans les approches cognitives comportementales puisqu'elles prescrivent les interventions. C'est d'ailleurs ce qui semble désormais désavantager ce type d'approche dans le champ sociojudiciaire postmoderne.

En effet, les études plus récentes sont assez critiques envers la prétendue supériorité des programmes de traitement d'orientation cognitive comportementale. Par exemple, la revue de littérature systématique de Vennard, Sugg et Hedderman (1997) montre que cette méthode ne produit pas d'effets différents de ceux des approches traditionnelles de type « casework ». Une étude récente a même indiqué que le taux de récidive des personnes soumises à cette approche était de $22,5 \%$ plus élevé que celui du groupe contrôle (Ong, Al-Attar, Roberts et Harsent, 2003). Une autre méta-analyse indique quant à elle que la méthode d'intervention spécifique n'explique que $15 \%$ de la variance dans la réussite d'une intervention. Les effets placébos comptent également pour $15 \%$, alors que les caractéristiques de la personne (incluant son réseau social et sa motivation) expliquent $40 \%$ de la réussite d'une intervention et, enfin, que la qualité de la relation thérapeutique compte pour 30 \% (Asay et Lambert, 1999). Ce constat est particulièrement intéressant, puisque depuis les débuts de la discipline du travail social, on insiste sur l'importance de la qualité de la relation thérapeutique comme outil de changement (Agllias, 2004; Parton, 1994).

Dans une société postmoderne, ce constat n'est pas étonnant. En effet, lorsque les normes et les sanctions sociales deviennent plus diffuses, les contradictions et les paradoxes sont plus nombreux, et il devient parfois difficile de choisir entre, par exemple, des valeurs individuelles et collectives. Le langage et la discussion prennent alors une importance capitale. C'est par le langage que les différents «sens » peuvent être partagés et que la «société » peut exister (Hugman, 2003). C'est donc à travers le dialogue que le travailleur social est en mesure de saisir ce qu'il y a d'unique, de contradictoire et d'incertain dans la situation du contrevenant, et 
qu'il peut alors développer « une nouvelle théorie du cas unique qui guidera ses actions » (Parton, 2000, p. 453). Dans un contexte postmoderne, le caractère « ambigu, incertain et contesté » (Parton, 1994, p. 18) du travail social devient soudain son principal atout. C'est cela qui lui assure un grand «potentiel de créativité, une liberté dans sa manière de penser et d'agir » (Parton, 2000, p. 46). Bref, c'est ce qui le distingue d'autres disciplines.

C'est aussi par le biais de la relation thérapeutique que le travailleur social arrive à rendre les contrôles «utiles ». Les contrôles seront utilisés tantôt comme ressource pour mobiliser le contrevenant, alors qu'à d'autres moments, ils serviront à faire exprimer un besoin ou une demande qui deviendra une cible de l'intervention (par exemple se loger, limiter sa consommation de substance toxicomaniaque, etc.). Ainsi, les contrôles « sont davantage un moyen s'inscrivant dans une stratégie qu'une tâche ou un but en soi » (Chauvenet et Orlic, 2002, p. 452). D'ailleurs, pour les travailleurs sociaux du champ sociojudiciaire, le cadre induit par la sanction pénale a l'avantage d'être clair. S'il dicte ce qui est attendu de la part du contrevenant, le cadre légal permet également au travailleur social de faire valoir les droits de ce dernier. C'est en exerçant ses droits sur le plan de l'accessibilité au logement, aux programmes d'aide à l'emploi (ou à l'assistance sociale), sur les plans familiaux ou autres, que le travailleur social arrive à aborder la question des faits reprochés et des obligations prononcées (Chauvenet et Orlic, 2002). C'est donc à l'intérieur de cette réciprocité des obligations et des droits que le travailleur social arrive à dépasser l'obstacle du contrôle et même « à faire reculer le contrôle au profit de l'aide » (Chauvenet et Orlic, 2002, p. 459).

Au-delà de l'importance accordée à l'établissement d'une relation thérapeutique, ce qui distingue également la discipline du travail social est son allégeance traditionnelle envers les populations appauvries, marginalisées ou discriminées. Or justement, ces populations sont celles qui sont les plus souvent pénalisées, et cela est vrai tant pour les taux d'arrestation, pour le nombre de poursuites que pour les condamnations (voir Reamur, 2004). Certains auteurs avancent même que le système pénal actuel sert à « gérer » ces populations qui ne sont plus soutenues par l'État-providence (Garland, 1996). En plus de dénoncer ces injustices, les travailleurs sociaux du champ sociojudiciaire proposent des modèles alternatifs à l'actuel système pénal. Une des alternatives qu'ils soutiennent est la justice réparatrice qui, basée sur la notion de discursivité, met en relation l'offenseur et l'offensé dans un contexte structuré et sécuritaire (Bradt et Bouverne-De Bie, 2009b). L'objectif de la justice réparatrice est de rendre compte des effets négatifs de l'infraction, de prévenir d'éventuelles infractions du même ordre et surtout de proposer une solution réparatrice à la victime (ou à la communauté). Ainsi, plutôt que d'opposer et de diviser, la justice réparatrice vise plutôt l'adoption d'une solution qui soit favorable aux deux parties opposées. Dans cette optique, la justice réparatrice permet également une redéfinition de la notion de responsabilité. En reconnaissant l'individualité du contrevenant, de la victime et de l'infraction, on arrive à mieux saisir « ce qui s'est passé ». La part de responsabilité reconnue par le contrevenant est d'autant plus porteuse de sens qu'elle tient compte de sa particularité, de sa trajectoire de vie, de son accès aux ressources et des contingences propres à son environnement social. Elle permet également aux victimes et aux communautés de reprendre du pouvoir d'agir dans la gestion des problèmes qui les concernent plutôt que d'avoir à s'en remettre aux institutions publiques (Charbonneau et Béliveau, 1999). Le rôle des travailleurs sociaux dans ce nouveau modèle de justice n'est pas de contrôler ou 
d'aider, mais plutôt d'agir en médiateur, rôle qu'ils remplissent par ailleurs déjà (voir Chouinard, Couturier et Lenoir, 2009). Si la justice réparatrice a fait son entrée au Canada, comme dans la plupart des pays occidentaux ${ }^{11}$, sous forme de projets pilotes, d'initiatives locales ou encore en étant destinée à des populations particulières (contrevenants adolescents ou Autochtones par exemple), il est intéressant de constater que ce modèle de justice fait désormais partie intégrante du système de justice pénale dans plusieurs pays européens (Bradt et Bouverne-De Bie, 2009b). Cela montre que le système de justice pénale n'est pas imperméable aux nouvelles idées et qu'il est possible de soutenir des initiatives en accord avec les valeurs centrales de la profession, soit celles de justice sociale, d'autodétermination et de respect de la dignité de la personne.

Ce rapide tour d'horizon de l'appropriation du champ sociojudiciaire par le travail social, de sa consolidation dans ce champ, de l'abandon du champ et de sa possible réappropriation ne saurait toutefois être complet sans que soient nommés les tensions et enjeux qui demeurent actuels. La première tension concerne la formation des travailleurs sociaux voulant œuvrer dans le champ sociojudiciaire: doit-elle être générique ou spécialisée? Aux États-Unis, le «forensic social work» est une spécialité qui s'accompagne de sa propre organisation nationale, de son code d'éthique et, habituellement, d'une formation de second cycle (voir Butters et Vaughan-Eden, 2011), alors qu'en Écosse, l'ensemble des travailleurs sociaux qui œuvrent au sein des «agences sociales » interviennent auprès des contrevenants (McNeill, Burns, Halliday, Hutton et Tata, 2009). Dans un contexte où les travailleurs sociaux ont perdu beaucoup de leur crédibilité et de leur pouvoir politique, soit en raison de leur allégeance traditionnelle envers les mesures réhabilitatives (McNeill et al., 2009) ou encore parce qu'il s'agit de la seule profession « d'aide » qui ne jouisse pas d'une spécialisation dans le champ (Green, Thorpe et Traupmann, 2005), il leur est difficile d'y affirmer une forte identité professionnelle. La spécialisation dans ce contexte peut alors paraître souhaitable, ce qui n'est d'ailleurs pas propre à ce champ (voir Randall et Kindiak, 2008). Une spécialisation pourrait aussi concourir au prestige du champ qui attirerait alors les travailleurs sociaux nouvellement formés. Toutefois, cette spécialisation pourrait nuire au caractère fluide et adaptatif de la profession qui, jusqu'à présent, lui a permis de «survivre » dans un environnement perçu comme hostile (Green et al., 2005; McNeill et al., 2009). Elle pourrait également exacerber le sentiment d'être « aliéné de la base » (Roberts et Brownell, 1999), risquant ainsi de fragiliser davantage les liens avec « la » discipline.

Un autre enjeu important est «l'instrumentalisation du communautaire » qui risque de réduire les initiatives qui émergent de la communauté pour leur préférer celles qui sont priorisées par les ministères de la Sécurité publique ou de la Justice. Toutefois, en raison du nombre limité d'études portant sur cette question, il est peut-être trop tôt pour se prononcer définitivement. Il se pourrait qu'à l'instar de leurs collègues écossais, les travailleurs sociaux québécois et canadiens montrent « une résignation et une adaptation aux orientations pénales actuelles accompagnée d'une mésadaptation et d'une révolte dans leurs pratiques » (McNeill et al., 2009, p. 435). Plus de cent ans au sein de ce champ les ont peut-être habitués à adopter

11. Royaume-Uni, États-Unis, Nouvelle-Zélande, Australie, Allemagne, Suisse et Belgique. 
les discours ambiants (et souvent contradictoires) du système pénal, sans que cela vienne modifier leurs pratiques, leurs valeurs et leurs croyances sur le terrain.

Il semble d'ailleurs que ce qui caractérise le mieux la longue alliance/mésalliance entre le travail social et le champ sociojudiciaire est cette irréductible croyance qu'il est possible de favoriser la justice sociale, l'autonomie, la dignité et le respect des personnes et des communautés à l'intérieur des contraintes prescrites par le système pénal. La présence des travailleurs sociaux dans ce champ, comme celle d'autres professionnels qui partagent cette croyance, a permis au système pénal canadien de ne pas sombrer dans les excès punitifs qui ont été observés dans d'autres pays occidentaux (O'Malley, 2004) et, ne serait-ce que pour cela, elle est plus que jamais nécessaire et justifiée.

Isabelle F.-Dufour

Doctorante en service social Boursière Canada Vanier 


\section{RÉFÉRENCES}

Ades, J., et B.E. Spiro (1985). «A social service lever for criminal justice sentencing », Social Service Review, 59(1), p. 95-106.

Agllias, K. (2004). « Women in corrections: A call to social work », Australian Social Work, 57(4), p. 331342.

Andrews, D.A. (1989). "Recidivism is predictable and can be influenced: Using risk assessments to reduce recidivism », Forum on Correction Research, 1(2), p. 11-18.

Andrews, D. A. et J. Bonta (1998). The psychology of criminal conduct, Ohio, Anderson Publishing.

Andrews, D. A., J. Bonta, P. Gendreau et F.T. Cullen (1990). «Does correctional treatment work? A clinically relevant and psychologically informed meta-analysis », Criminology, 28(3), p. 369-404.

Aranguiz, M. et J.-M. Fecteau (1998). "Le problème historique de la pauvreté extrême et de l'errance à Montréal depuis la fin du XIX ${ }^{\mathrm{e}}$ siècle », Nouvelles pratiques sociales, vol. 11, $\mathrm{n}^{0} 1$, p. 83-98.

Asay, T.P. et M. J. Lambert (1999). «The empirical case for the common factors in therapy », dans M.A. Hubble, B.L. Duncan et S.D. Miller (dir.), The heart and soul of change. What works in therapy?, Washington, American Psychological Association, p. 23-55.

Barry, M. (2007). « Listening and learning: The reciprocal relationship between worker and client », The Journal of Community and Criminal Justice, 54(4), p. 407-422.

Bradt, L. et M. Bouverne-De Bie (2009a). «Social work and shift from "Welfare" to "Justice", British Journal of Social Work, 39, p. 113-127.

Bradt, L. et M. Bouverne-De Bie (2009b). "Victim-offender mediation as a social work practice », International Social Work, 52(2), p. 181-193.

Burford, G. et P. Adams (2004). «Restorative justice, responsive regulation and social work », Journal of Sociology and Social Welfare, 31(1), p. 7-25.

Burnett, R. (2002). "The case for counselling as a method for working with offenders », Vista, 7(3), p. 216-226.

Burnett, R. (2004). " One-to-one ways of promoting desistance: in search of an evidence base », dans R. Burnett et C. Roberts, What works in probation and youth justice: Developing evidence-based practice, Cullompton, Willan, p. 180-197.

Butters, R.P. et V. Vaughan-Eden (2011). «The ethics of practicing forensic social work », Journal of Forensic Social Work, 1, p. 61-72.

Chaiklin, H. (2007). « Epilogue: Social work and criminal justice?», dans D.W. Springler et A. R. Roberts (dir.), Handbook of forensic mental health with victims and offenders: Assessment, treatment and research, New York, Springer Publishing Company, p. 573-586.

Charbonneau, S. et D. Béliveau (1999). « Un exemple de justice réparatrice au Québec: La médiation et les organismes de justice alternative », Criminologie, vol. $32, n^{0} 1$, p. 57-77.

Chauvenet, A. et F. Orlic (2002). "Sens de la peine et contraintes en milieu ouvert et en prison », Déviance et Société, vol. 26, $\mathrm{n}^{\circ}$ 4, p. 443-461. 
Chouinard, I., Y. Couturier et Y. Lenoir (2009). « Pratique de médiation ou pratique médiatrice? La médiation comme cadre d'analyse de la pratique professionnelle des travailleurs sociaux », Nouvelles pratiques sociales, vol. $21, \mathrm{n}^{\circ} 2$, p. 31-45.

Cohen, S. (1985). Visions of social control. Crime, punishment and classification, Cambridge, MA, Polity Press.

Commission royale d'enquête sur le système pénal du Canada (rapport Archambault) (1938). Rapport de la commission royale d'enquête sur le système pénal du Canada, Ottawa.

Donzelot, J. (1980). The policing of families: Welfare versus the State, London, UK, Hutchinson.

Dufresne, M. et R. Hastings (2003). « La restructuration de l'action dans le champ de la régulation sociopénale de la jeunesse au Québec », Déviance et Société, vol. 27, nº 4, p. 413-428.

Ekstedt, J.W., et C.T. Griffiths (1988). Corrections in Canada: Policy and practice ( $2^{\mathrm{e}}$ éd.), Toronto, Butterworths.

Favreau, L. (2000). «Le travail social au Québec (1960-2000): 40 ans de transformation d'une profession », Nouvelles pratiques sociales, vol. 13, $n^{0} 1$, p. 27-47.

Fédération internationale des travailleurs sociaux (2000). « Définition du travail social ». [http://www.ifsw.org/p38000376.html.]

Foucault, M. (1975). Surveiller et punir: Naissance de la prison, Paris, Gallimard.

Franklin, D. L. (1990). « The cycles of social work practices: Social action vs. individual interest », Journal of Progressive Human Services, 1(12), p. 59-80.

Garland, D. (1985). Punishment and welfare: A history of penal strategies, Aldershot, Angleterre, Gower.

Garland, D. (1996). «The limits of the sovereign state: Strategies of crime control in contemporary society », British Journal of Criminology, 36(4), p. 445-471.

Gendreau, P., T. Little et C. Groggin (1996). « A meta-analysis of the predictors of adult recidivism: What works!», Criminology, 34, p. 575-607.

Goff, C. (2003). Criminal justice in Canada, Scarborough, Thompson Nelson.

Green, G., J. Thorpe et M. Traupmann (2005). « The sprawling thicket: Knowledge and specialisation in forensic social work », Australian Social Work, 58(2), p. 142-153.

Gumz, E.J. (2004). «American social work, corrections and restorative justice: An appraisal», International Journal of Offender Therapy and Comparative Criminology, 48(4), p. 449-460.

Hugman, R. (2003). "Professional values and ethics in social work: Reconsidering postmodernism?», British Journal of Social Work, 33(8), p. 1025-1041.

Hussey, F. A. et D. E. Duffee (1980). Probation, parole and community field services. Policy, structure and process, New York, Harper \& Row Publishers.

Kaminski, D. (2006). « Un nouveau sujet de droit pénal?», dans F. Digneffe et T. Moreau (dir.), La responsabilité et la responsabilisation dans la justice pénale, Bruxelles, Lacier et De Boeck, p. 323342. 
Kidman, J. (1947). The Canadian prison: The story of a tragedy, Toronto, Ryerson Press.

Lancaster, E. et J. Lumb (2006). «The assessment of risk in the National probation service of England and Wales », Journal of Social Work, 6(3), p. 275-291.

Landreville, P. (1986). « Évolution théorique en criminologie. L'histoire d'un cheminement », Criminologie, vol. $19, \mathrm{n}^{\circ} 1$, p. $11-31$.

Laplante, P. (1989). Prison et ordre social au Québec, Ottawa, Les Presses de l'Université d'Ottawa.

Lipton, D. S., D. Thornton, J. McGuire, F. J. Porporino et C. R. Hollin (2000). « Program accreditation and correctional treatment », Substance Use and Misuse, 35(12-14), p. 1705-1734.

Mair, G. (1997). "Community penalties and the probation service », dans M. Maguire, R. D. Morgan et R. Reiner (dir.), The Oxford Handbook of Criminology ( $2^{\mathrm{e}}$ éd.), Oxford, Clarendon, p. 1195-1232.

Martinson, R. (1974). «What Works? Question and answers about prison reform », Public Interest, 25, p. 22-54.

Maruna, S. (2001). Making good. How ex-convicts reform and rebuild their lives, Washington, American Psychological Association.

Maschi, T., N. M. Violette, N. Scotto Rosato et J. Ristow (2009). « Juvenile justice and social work », dans T. Maschi, C. Bradley et K. Ward (dir.), Forensic social work: Psychosocial and legal issues in diverse practice settings, New York, Springer Publishing Company, p. 231-254.

Mauruto, P. et K. Hannah-Moffat (2006). « Assembling risk and the restructuring of penal control », British Journal of Criminology, 46, p. 438-454.

Mayer, R. (2002). Évolution des pratiques en service social, Montréal, Gaëtan Morin Éditeur.

McNeill, F., N. Burns, S. Halliday, N. Hutton et C. Tata (2009). « Risk, responsibility and reconfiguration. Penal adaptation and misadaptation », Punishment and Society, 11(4), p. 419-442.

Normandeau, A. (1979). « Faut-il transformer ou abolir la probation? », Criminologie, vol. 12, $\mathrm{n}^{\circ}$ 2, p. 89105.

Ohlin, L.E., H. Piven et M. Pappenfort (1956). « Major dilemmas of the social worker in probation and parole », National Probation and Parole Association Journal, 3, p. 211-225.

O'Malley, P. (2004). "The uncertain promise of risk», The Australian and New Zealand Journal of Criminology, 37(3), p. 323-343.

Ong, G., Z. Al-Attar, C. Roberts et L. Harsent (2003). Think First: An accredited community-based cognitive-behavioural programme in England and Wales. Findings from the prospective evaluation in three probation areas, London, Home Office.

Parton, N. (1994). «"Problematics of government”, (post)modernity and social work », British Journal of Social Work, 24, p. 9-32.

Parton, N. (2000). "Some toughts on the relationship between theory and practice in and for social work », British Journal of Social Work, 30, p. 449-463.

Philip, M. (1979). « Notes on the form of knowledge in social work », Sociological Review, 27(1), p. 83111. 
Poupart, J. (2004). «L'institutionnalisation de la criminologie au Québec. Une lecture sociopolitique », Criminologie, vol. $37, n^{0} 1$, p. 71-105.

Randall, G.E. et D. H. Kindiak (2008). « Deprofessionalization or postprofessionalization? Reflections on the state of social work as a profession », Social Work in Health Care, 47(4), p. 341-354.

Reamur, F. G. (2004). « Social work and criminal justice: The uneasy alliance », Journal of Religion and Spirituality in Social Work, 23(1-2), p. 213-231.

Richmond, M. E. (1917). Social diagnosis, New York, Russell Sage Foundation.

Roberts, A.R. et P. Brownell (1999). «A century of forensic social work: Bringing the past to present », Social Work, 44(4), p. 1-17.

Treger, H. (1983). « Social work in the justice system: An overview », dans A. R. Roberts (dir.), Social work in juvenile and criminal justice settings, Springfield, Charles C. Thomas, p. 7-17.

Vaillancourt, Y. (2002). «Le modèle québécois de politiques sociales et ses interfaces avec l'union sociale canadienne », Enjeux publics, vol. 3, n 2, p. 1-52.

Vennard, J., D. Sugg et C. Hedderman (1997). The use of cognitive-behavioural approaches with offenders: Messages from the research, London, Home Office.

Young, D.S. et S.W. LoMonaco (2001). «Incorporating content on offenders and corrections into social work curricula », Journal of Social Work Education, 37(3), p. 475-489. 